\title{
Atomic Level Mechanisms of Solid-State Dewetting in Thin Metal Films Deposited on Silicon (100) Substrates
}

\author{
A.M. Thron, ${ }^{*}$ P. Greene, ${ }^{* *}$ Kai Liu, ${ }^{* *}$ K. van Benthem, ${ }^{*}$ \\ *Department of Chemical Engineering and Materials Science, One Shields Avenue, \\ University of California, Davis, Davis CA 95616 \\ **Department of Physics, One Shields Avenue, University of California, Davis, Davis \\ CA 95616
}

Solid-state dewetting is a diffusion driven process in which the nucleation and growth of voids in a thin film lowers the free energy of the thin film and its interface to the substrate. Observations of void formation in thin metal films, well below their bulk critical points, were previously [1,2], and the reported results are consistent with Cahn's critical point wetting theory [3]. For the atomic-scale determination of structural and compositional gradients across the interface we use aberration-corrected Scanning Transmission Electron Microscopy (STEM) and Electron Energy-loss Spectroscopy (EELS). Dewetting processes are initiated by heating the cross-sectional TEM sample during High Resolution Transmission Electron Microscopy (HRTEM) observation.

Thin nickel films with nominal thicknesses ranging from 5-20nm were deposited on silicon (100) substrates by DC magnetron sputtering. Dilute hydrofluoric acid was used to remove any native oxide layer on the silicon substrate prior to deposition. Sputtering of nominally $20 \mathrm{~nm}$ and $5 \mathrm{~nm}$ thick nickel films was carried out at deposition rates of 2.7 $\mathrm{nm} / \mathrm{sec}$ and $11 \mathrm{~nm} / \mathrm{sec}$, respectfully. Sputtering occurred at room temperature and a base pressure of $1.8 \times 10^{-8}$ torr. Cross-sectional TEM specimens of the as-deposited films were prepared by standard cutting, grinding, dimpling and ion-milling techniques [4].

HRTEM micrographs of the as-deposited films show two poly-crystalline layers separated from the substrate by an amorphous interlayer of up to $1.8 \mathrm{~nm}$ in thickness (see Figure. 1a). The first layer exhibits crystal grains $15-76 \mathrm{~nm}$ wide and roughly $5 \mathrm{~nm}$ thick. The second layer consists of significantly smaller grains. Selected area diffraction patterns confirm the polycrystalline crystal structure of the Ni film with indications of a limited number of preferred orientation relationships, i.e. in-plane rotations (Figure 1b). Preliminary data show strain contrast at grain boundaries between the first and second crystalline layers and may suggest the presence of misfit dislocations and twinning.

Previous studies report that the first few monolayers during deposition of nickel at room temperature form a nickel silicide $\left(\mathrm{Ni}_{2} \mathrm{Si}\right)$ layer, before pure nickel films continue to grow [6]. STEM-EELS measurements confirm the presence of an initial silicide-like layer. Near-edge fine structures of the $\mathrm{Si} \mathrm{L}_{2,3}$ and $\mathrm{Ni} \mathrm{L}_{2,3}$ ionization edges are currently being analyzed for a more detailed analysis of local compositional gradients.

Preliminary in-situ heating experiments demonstrate that dewetting of thin metal films occurs at reasonable rates for real-time HRTEM acquisition (Figure. 2). First results about dynamic dewetting processes in the $\mathrm{Ni} / \mathrm{Si}$ system will be reported during the presentation. 

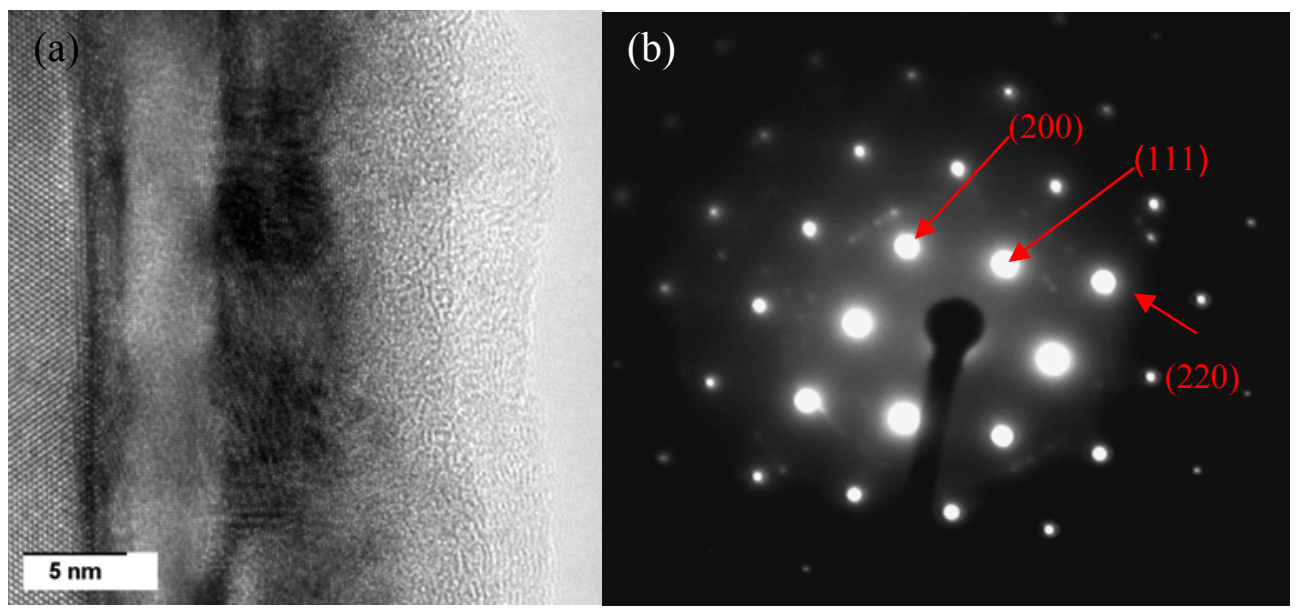

Figure 1: (a) Cross-sectional HRTEM micrograph of the as-deposited nickel film. (b) SAD pattern from an area similar to the micrograph of the interface configuration with the silicon substrate in $<110>$ zone-axis orientation.
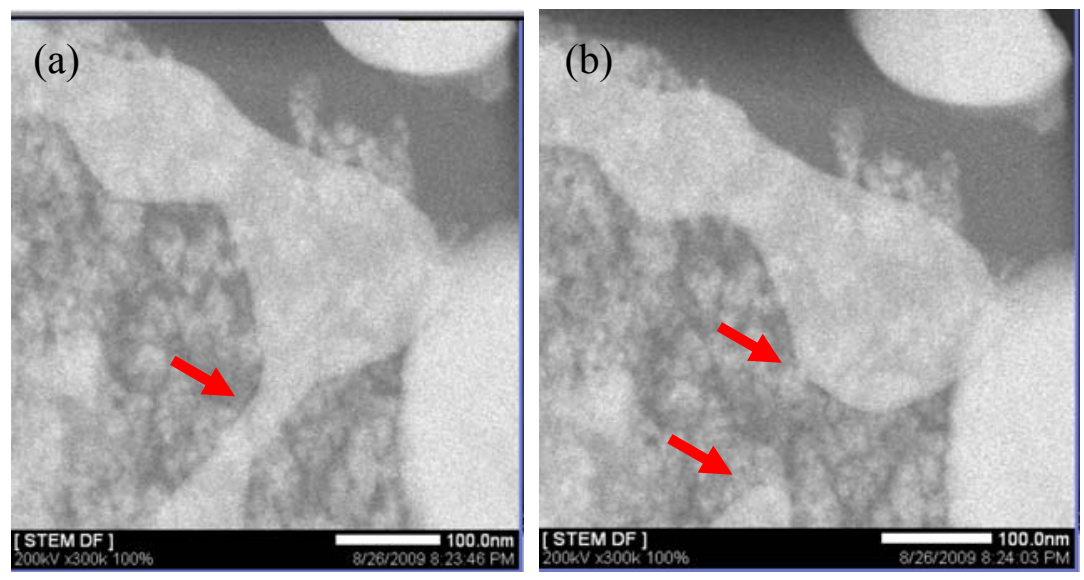

Figure 2: Evidence of void formation during preliminary, in-situ dewetting experiments of an as-deposited thin bi-layer of $\mathrm{Cu}$ and $\mathrm{Ni}$ on a $\mathrm{Si}(100)$ substrate in plan-view. The time elapsed between Figure (a) and Figure (b) is approximately 17 seconds.

[1] E. Jiran, C.V. Thompson, Thin Solid Films 208, 23 (1992).

[2] J. Peterson, S.G. Mayr, J App. Phys 103, 023520 (2008).

[3] J.W. Cahn, J. Chem. Phys. 66, 3667 (1977).

[4] A. Strecker et. al., Z. Metallkd. 94, 290 (2003).

[5] A.L. Giermann, C.V. Thompson, App. Phys. Lett. 86, 121903 (2005).

[6] T. Nishimura et. al., Surf. Sci. 588, 71 (2005).

[7] The authors acknowledge financial support through start-up funds from the University of California at Davis. Assistance during TEM sample preparation by Mr. Seth Weil is appreciated. Parts of this research were carried out at the National Center for Electron Microscopy (NCEM) at Lawrence Berkeley National Laboratory. 